\title{
Former des enseignants de français pour les nouvelles humanités numériques : enjeux épistémologiques et empiriques
}

Monique Lebrun

\section{(2) OpenEdition \\ Journals \\ Édition électronique \\ URL : http://journals.openedition.org/trema/3319 \\ DOI : 10.4000/trema.3319 \\ ISSN : 2107-0997 \\ Éditeur \\ Faculté d'Éducation de l'université de Montpellier}

Édition imprimée

Date de publication : 1 mai 2015

Pagination : 68 - 77

ISSN : 1167-315X

\section{Référence électronique}

Monique Lebrun, «Former des enseignants de français pour les nouvelles humanités numériques : enjeux épistémologiques et empiriques », Tréma [En ligne], 43 | 2015, mis en ligne le 25 juin 2015, consulté le 19 avril 2019. URL : http://journals.openedition.org/trema/3319; DOI : 10.4000/ trema.3319

Ce document a été généré automatiquement le 19 avril 2019.

Trema 


\title{
Former des enseignants de français pour les nouvelles humanités numériques : enjeux épistémologiques et empiriques
}

\author{
Monique Lebrun
}

\section{Introduction}

1 Il est difficile, en ces jours où l'outil numérique et les réseaux divers qu'il engendre s'imposent partout dans les sphères sociale et personnelle, de voir comment former l'enseignant de français, et plus spécifiquement l'enseignant de lettres. Comment lui apprendre à sauvegarder l'héritage culturel et littéraire qu'on lui a transmis en le conjuguant aux avancées de la technologie moderne? Les repères et les codes de la formation des maîtres traditionnelle semblent ébranlés depuis l'injonction des programmes officiels de tenir compte des TIC dans toutes les disciplines.

2 Nous explorerons cette question en parlant tout d'abord du concept d'humanités numériques, qui ne vient pas remplacer celui des humanités traditionnelles, mais le compléter. Suivront, en deuxième partie, quelques précisions sur le concept de littératie médiatique multimodale et ses applications dans le champ de la formation des enseignants de français. Nous donnerons en troisième et dernière partie un éclairage plus précis sur cette question en évoquant la rentabilité du modèle TPACK.

\section{Des humanités classiques aux humanités numériques}

3 La prolifération des savoirs numériques propre à notre époque nous incite à nous interroger sur ce qui doit faire l'essentiel de notre culture, sur la notion même 
d'« humanités », en fait. Pour Lankshear et Knobel (2003), on entre désormais dans une ère de questionnement sur ce qui doit compter comme savoir, sur la façon d'y accéder, et même sur la façon de l'utiliser. En somme, aujourd'hui, nous disent les auteurs, « savoir, c'est performer» (knowing as an habilety to perform, 2003, 173) et c'est être capable d'utiliser des stratégies telles que la cueillette de données, leur transformation et leur dissémination dans un but de communication inséré dans un univers social déterminé.

4 L'arrivée des TIC en éducation en a inquiété plusieurs, qui y ont vu une dérive fonctionnaliste et une orientation vers l'économie de marché, au détriment de la formation de l'individu dans une perspective humaniste. On se retrouve ici dans la distinction durkheimienne entre le profane et le sacré. Le sacré se réfère à des formes spécifiques de relation au savoir et aux obligations sociales et discursives que cette relation requiert. Le profane, pour sa part, se réfère aux demandes contextuelles et aux contraintes du système économique. Adopter ce genre de dichotomie, en assimilant les TIC au profane, c'est refuser de reconnaitre que les TIC sont fondatrices d'un nouvel humanisme, l'humanisme numérique et non d'une sous-culture propre aux informaticiens et aux adolescents de type geek. Nous parlerons pour notre part de la capacité qu'ont les TIC, couplées à la pédagogie et aux contenus disciplinaires, de transformer les savoirs et l'apprentissage de ces savoirs, ce qui est tout de même crucial tant pour la formation des enseignants que pour leurs élèves.

5 Les nouvelles technologies constituent un formidable instrument de documentation et d'échange, mais elles peuvent aussi être un espace de création, voire de réflexion, qui revivifie la culture humaniste traditionnelle. Il faut suivre à cet égard le raisonnement de Douehi (2011), pour lequel l'humanisme numérique est le résultat quelque peu enivrant et affolant à la fois de la confluence d'un héritage culturel riche et foisonnant, d'une part, et des avancées de technologies numériques qui permettent vraiment de réaliser le « village global ", d'autre part.

6 L'expression «humanités numériques" est née en 2006 et connaît depuis un incontestable succès, même si, comme le rappelle Mounier (2012), on peut s'interroger sur les paradigmes intellectuels qui fondent ces nouvelles humanités et sur la structuration du champ. C'est entre autres le cas de la place laissée à l'interprétation dans ces recherches qui semblent tout entière dirigées par les données; ou encore, de la place démesurée de la littératie d'information dans un environnement web 1.0 ou 2.0 qui semble n'avoir été créé que pour la produire, la disséminer et la consommer.

Plusieurs auteurs, dont Liu (2012), Le Deuff et Berra (2012) ont insisté sur le potentiel transdisciplinaire du numérique, qui lui permet de bâtir des ponts entre les disciplines et, éventuellement, d'amorcer une critique culturelle des humanités numériques.

8 On peut parler chez les jeunes d'une «culture numérique ", qui fait partie de la culture médiatique contemporaine, cette dernière désignant au sens large une connaissance des médias audiovisuels, imprimés et numériques (Tabary-Bolka, 2009). Cette culture numérique, construite la plupart du temps hors de l'école, a ses valeurs (ex : expression de soi, désir de partage de documents et de «trouvailles»), ses connaissances (ex: meilleurs sites d'échange de photos, techniques pour construire des multitextes) et ses pratiques propres (ex : usage du blog et des abréviations, création de contenus nouveaux par remixage ou hybridation - appelée aussi « redocumentarisation »).

9 Le « texte » numérique est donc hybride, et c'est là sa grande originalité par rapport au texte traditionnel. Il suppose des relations de jonction, de fusion, de transposition entre 
les produits numériques, et ces relations sont parfois tellement complexes qu'elles exigent un apprentissage, d'abord pour comprendre de pareils textes, et ensuite, pour en produire.

\section{La littératie médiatique multimodale et ses fondements épistémologiques et empiriques}

10 Lorsqu'il est question des nouvelles technologies appliquées à l'enseignement des langues , et, dans une moindre mesure, des disciplines de sciences humaines, on parle de littératie médiatique multimodale, Celle-ci s'intéresse à la compréhension et la production d'écrits sur des supports variés, dont des supports numériques, intégrant à des degrés divers, le texte, l'image et le son (Lebrun, Lacelle et Boutin, 2012). C'est donc une littératie qui combine le traditionnel et le numérique et qui conjugue différents modes sémiotiques (iconiques, linguistiques et auditifs), souvent sur le même support (d'où la notion de «multitexte »), dans la même production (une séquence vidéo, par exemple, comprend images animées et sons, les deux étant livrés conjointement et est donc un multitexte). Le domaine est devenu interdisciplinaire, sollicitant la collaboration de spécialistes de la littératie classique, de la culture, de l'éducation aux médias, de l'interaction humainordinateur et des nouvelles technologies. Les politiques éducatives ont commencé à s'y intéresser, voyant poindre une nouvelle définition de l'homme alphabétisé. Une littératie médiatique bien conçue, épistémologiquement, suppose une posture critique de la part de ses usagers, c'est-à-dire une tendance à s'interroger sur ce qui constitue la nature ou le message du multitexte.

11 La littératie médiatique multimodale semble abolir, dit Buckingham (2003), la distinction entre lecteur et scripteur. En lecture multimodale, le lecteur n'est pas guidé uniquement par le texte et sa linéarité, car le seul texte possible devient celui qu'il choisit d'» écrire ». Ce qui est sous-entendu ici, c'est l'interactivité suscitée par la lecture des textes multimodaux et facilitée par la convivialité du support numérique. L'idée de multimodalité n'est pas nouvelle, ainsi que l'ont précisé Kress $(2009,2010)$ et Jewit (2009); ce qui l'est, c'est le relais et la distribution des messages via une pluralité grandissante de médias.

12 Les bases empiriques de la littératie médiatique multimodale ne sont pas encore solidement établies, ainsi que l'ont remarqué plusieurs chercheurs. Pareille entreprise doit passer par l'analyse de la production médiatique multimodale (Walsh, 2008; Kress, 2003), et plus particulièrement par l'analyse des différents systèmes sémiotiques mis en oeuvre et celle des processus de construction du sens propres aux multitextes. Il faut donc que les expériences sur le terrain se multiplient pour donner un éclairage plus pragmatique aux possibilités de la littératie médiatique multimodale.

13 Le contexte d'expansion exponentielle des nouveaux médias offre aux enseignants l'occasion d'ajuster leur rôle professionnel, d'enrichir leurs connaissances et d'acquérir de nouvelles valeurs, plus spécifiquement en ce qui concerne l'apprentissage (ce qu'il est important d'apprendre et comment le faire), comme le disent Koehler et Mishra (2005). Ils doivent repérer les élèves habiles dans les nouveaux médias, et non seulement ceux qui lisent et écrivent bien. L'approche pédagogique a aussi son importance, les nouvelles littératies n'étant pas solubles dans la pédagogie magistro-centrée. La conversion des enseignants aux pratiques de littératie en classe passe par l'auto-apprentissage à l'aide de 
guides de leur milieu de travail, et, s'ils sont en formation, par des curricula renouvelés et sensibles aux apports du numérique dans les champs disciplinaires.

Quelques dangers persistent, au plan épistémologique, dont celui de confondre la littératie médiatique multimodale avec un simple usage des TIC. On continue encore, en formation des enseignants, à avoir une perspective uniquement instrumentale par rapport aux nouvelles technologies, en hypertrophiant l'importance à leur donner comme outil. C'est entre autres le cas de tout un courant pour lequel il faut initier les enseignants aux TIC sans lier celles-ci à des contenus particuliers et sans voir que les TIC, justement, peuvent être à la base d'un renouveau dans les didactiques disciplinaires. On le voit dans les opinions de Karsenti, Péraya et Viens (2002), qui lient TIC et nouvelle pédagogie, mais oublient au tournant de parler de contenus disciplinaires spécifiques. Leur option, c'est la techno-pédagogie, et cela se sent dans leurs propos. Ils sont très vagues sur les « savoirs » et se confinent à affirmer que l'usage des TIC devrait permettre aux enseignants d'aider leurs élèves à les construire.

Il en est aussi qui traitent de contenus TIC reliés plus ou moins à leur discipline, mais en se concentrant sur cette orientation techno-pédagogique. Ainsi, quand Rey et Beaudet (2011) parlent de la nécessité de former des stagiaires à l'enseignement à la lecture et à l'écriture en hypertexte, elles ne vont pas assez loin, à notre avis. Les activités des stagiaires qu'elles décrivent (ex : transmettre un plan de cours par Internet, donner des sitographies aux élèves, donner des exercices de traitement de texte, etc) sont non seulement très magistro-centrées, mais également très conservatrices dans leur essence, puisque le Web y est vu uniquement comme un outil, et, qui plus est, un outil ponctuel dans la démarche didactique. Il y a certes un début à tout et nous savons que le maniement des TIC fait partie du Référentiel de compétences professionnelles en France (Boissinot, 2010). Une enquête de l'OCDE dans onze pays (Enochsson et Riza, 2009, 6) a cependant démontré que les enseignants en formation n'intègrent pas suffisamment les nouvelles technologies dans leurs pratiques pédagogiques et qu'il faudrait les former à le faire par divers moyens, par exemple, «intégrer les TIC de manière usuelle dans la matière enseignée ou lors des stages, connaître le monde technologique des élèves ».

\section{Le modèle TPACK et le développement pro- fessionnel en littératie médiatique multimodale chez les stagiaires à l'enseignement}

16 Le concept de «design » ou orchestration des modes de représentation (linguistiques, iconiques, auditifs, gestuels) est incontournable en littératie médiatique multimodale, comme l'ont souligné Kress (2003), Jewitt et Kress (2003) et le New London Group (2000). Les enseignants doivent y performer, pour systématiser les apprentissages, relier les littératies nouvelles à la littératie traditionnelle.

17 Les chercheurs de littératie médiatique multimodale anglo-saxons se sont beaucoup penchés sur la question, mais ceux de la francophonie tardent encore à le faire. Il faut ici bien distinguer l'usage des TIC en général de celui de la littératie médiatique multimodale. On l'a dit précédemment, les travaux sur l'usage des TIC à l'école sont légion et ils font surtout référence à l'ordinateur comme outil (ex: rechercher de l'information; créer un tableau d'entrée de données, coller des images à un texte). En littératie médiatique multimodale, il s'agit d'aller plus loin et d'intégrer véritablement les 
modes de représentation sémiotiques divers dans une démarche qui vise le meilleur apprentissage de la lecture et de l'écriture.

Des ateliers de formation pour enseignants qui ne se baseraient que sur le développement d'habiletés technologiques et d'exercices décontextualisés à partir de logiciels rateraient l'occasion de donner aux enseignants une formation adéquate sur toutes les possibilités des nouvelles littératies.

Dans leur recherche sur les apprentissages en littératie reliés aux TIC dans une vingtaine de classe, Lankshear et Knobel (2003) ont trouvé que les enseignants réduisaient les propriétés de l'outil technologique à des exercices très scolaires, au détriment d'authentiques pratiques sociales. Ils ont expliqué cette attitude par le manque d'expérience des enseignants en TIC, ce qui les a entraînés à se centrer uniquement sur l'aspect fonctionnel de l'outil numérique, mettant celui-ci au service de pratiques traditionnelles. Ces enseignants se sont perçus, disent-ils, comme des guides technologiques, au lieu de considérer le contexte plus large des possibilités de l'outil dans le développement de la littératie. On était loin ici de la création de sites web et de l'écriture spontanée de fanfiction que pratiquent plusieurs élèves et qui sont d'authentiques pratiques multimodales. Pour que l'école s'adapte, concluent les chercheurs, les enseignants doivent tout d'abord intégrer les nouvelles littératies dans leur vie personnelle (comme leurs élèves). Ils seront ensuite plus tentés de changer leurs façons d'enseigner.

Le changement de paradigme prend toutefois du temps et fait partie du développement professionnel des enseignants. Ceux-ci ont besoin de soutien technique et didactique, de temps de réflexion, d'échanges avec leurs pairs et de modèles efficaces de scénarios utilisant la nouvelle littératie. Les chercheurs Koehler et Mishra (2005) en savent quelque chose. Ils ont suivi durant cinq ans le développement professionnel d'enseignants utilisant les technologies dans leurs cours universitaires. Ils ont créé un cadre conceptuel (le modèle TPACK) pour aller au-delà de la dichotomie perçue entre les savoirs technologiques et les savoirs disciplinaires, qui inhibe le développement professionnel. Leur représentation conceptuelle apparaît ci-dessous. 


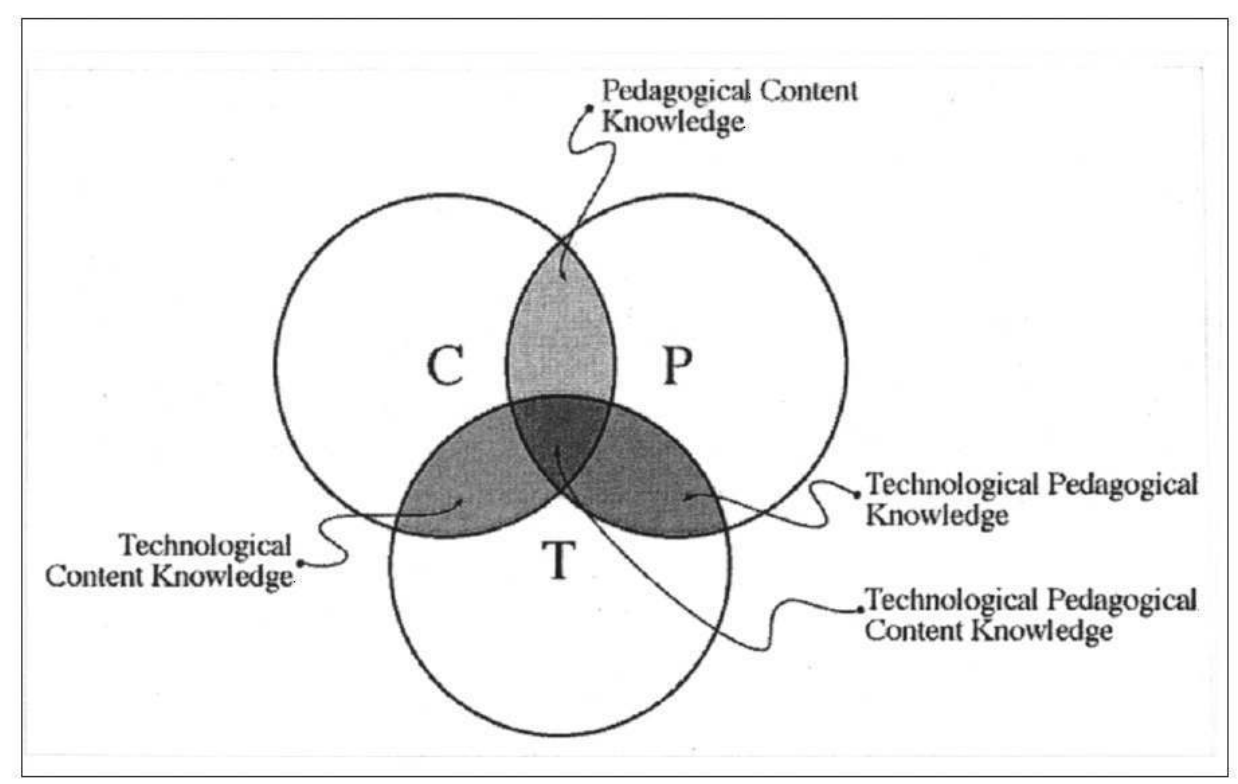

Figure 1. The components of Technological Pedagogical Content Knowledge.

Les composantes du modèle TPAK (Source : Koehler et Mishra, 2005, p. 133)

$21 \mathrm{Au}$ cœur de la figure apparaissent trois zones de savoir : le contenu, la pédagogie et la technologie. La zone $\mathrm{C}$ représente le contenu qui doit être enseigné et appris; il s'agit d'une discipline scolaire spécifique. La zone $\mathrm{T}$ a trait aux technologies, que celles-ci soient nouvelles (ordinateur, Internet, vidéo numérique) ou anciennes (tableau noir, livres). La zone $\mathrm{P}$ est celle de la pédagogie et comprend les pratiques, les processus, les stratégies, les méthodes d'enseignement et d'apprentissage, de même que les objectifs éducatifs et l'évaluation.

Il est important de considérer l'aire de recoupement et de chevauchement de ces trois zones. L'aire de fusion des zones $\mathrm{P}$ et $\mathrm{C}$ nous permet de parler de «savoir à contenu pédagogique »; là se trouvent les représentations de concepts, les techniques pédagogiques et la connaissance des savoirs antérieurs des élèves, entre autres. L'aire de fusion des zones $\mathrm{T}$ et $\mathrm{C}$ permet de construire le concept de "savoir à contenu technique ", qui couvre les savoirs enseignants sur la façon dont une discipline est transformée par la technologie (ex: la simulation d'un procès pour étudier le roman policier). L'aire de fusion des zones $\mathrm{T}$ et $\mathrm{P}$ peut être décrite comme celle du "savoir techno-pédagogique ", ou connaissances sur la façon dont les technologies permettent d'atteindre des buts éducatifs (ex : la collaboration). L'aire la plus importante pour notre propos se situe à la jonction des trois aires de fusion précédentes et est nommée par Koehler et Mishra (2005) "savoir sur le contenu, la pédagogie et la technique », zone d'intégration dynamique du savoir et de la techno-pédagogie, qui suppose une approche par "design pédagogique » permettant de travailler avec les nouvelles technologies dans un contexte réel d'apprentissage et surtout, dans une discipline donnée (ce qui est le propre de la littératie médiatique multimodale pour ce qui est des langues).

Se basant sur ce schéma conceptuel, Koehler et Mishra (2005) ont ensuite tenté de voir en quoi les enseignants de leur échantillon utilisaient la technologie pour améliorer 
l'enseignement de leur discipline et développaient ainsi leur savoir professionnel, par exemple en construisant un cours en ligne, ou un site web, ou encore en produisant des vidéos numériques d'apprentissage disciplinaire. À leur avis, l'enseignement ciblé que leurs sujets ont reçu lors de séminaires universitaires leur a permis de vivre une véritable intégration aux nouvelles littératies, favorisant l'engagement actif des élèves. Ils ne sont toutefois pas allés vérifier l'impact du cours sur leurs pratiques en salle de classe. Pour eux, l'application du modèle TPACK requiert un changement curriculaire qui se concrétise en une approche qu'ils appellent l'apprentissage technologique par le design pédagogique (learning technologies by design), qui est une approche constructiviste centrée sur l'interaction.

Miller (2007) est l'une des plus ferventes adeptes du TPACK pour la formation des enseignants. Selon elle, les pratiques de littératie multimodale permettent de recadrer les objectifs pédagogiques en les liant aux pratiques de littératie extra-scolaires des élèves par le biais d'activités planifiées et vécues dans des espaces sociaux qui engagent l'élève comme être global et transforment son apprentissage. L'auteure travaille à la formation et au perfectionnement des enseignants d'anglais langue maternelle. Elle désire revitaliser les pratiques qui ont cours chez ces enseignants. Elle s'est spécialisée dans la création de vidéos numériques éducatives avec ceux-ci, en lien avec des séminaires de développement professionnel incluant enseignants en exercice et en formation. Elle les a initiés aux divers modes sémiotiques, à la rédaction d'un script, au tournage, au montage, à l'intégration de la vidéo à l'ordinateur à l'aide de programmes simples (iMovie et MovieMaker). Pour elle, la production vidéo constitue la quintessence de la littératie médiatique multimodale, car elle permet l'orchestration des différents modes sémiotiques. Elle peut également couvrir tous les aspects d'un programme de langue maternelle (ex: vidéos sur le théâtre, sur la poésie, sur la création d'une pub, sur un mouvement littéraire, sur un bulletin de nouvelles, etc). En plus de produire des vidéos numériques en équipes, les enseignants de son séminaire ont participé à des discussions métacognitives sur le processus même de la production, ont incité leurs propres élèves à s'engager dans la production de vidéos et sont revenus en séminaire faire part de la réaction de ceux-ci. Cela a permis à tous les participants de s'interroger sur les finalités de l'exercice pour l'apprentissage des savoirs reliés à la langue et sur les facilités qu'offre le milieu scolaire pour ce faire.

On retrouve chez Hofer et Harris (2010) une étude comparative des activités offertes aux enseignants en service et aux stagiaires d'anglais langue maternelle selon le modèle TPACK. Ils ont noté que ces derniers avaient besoin davantage d'étayage dans toutes les activités proposées, particulièrement en raison de leurs lacunes en planification et en connaissance du terrain. Des cours spécifiques de planification intégrant contenu disciplinaire et TIC ont été mis à leur programme de formation. Les activités de littératie médiatique qu'ils ont eu à vivre se basaient sur une taxonomie des processus de lecture et d'écriture. Les diverses technologies utilisables ont été touchées et la formation a permis également des échanges sur l'apport de ces technologies à la formation. Ainsi les étudiants-maîtres, une fois la planification de leurs activités achevée, devaient justifier leurs choix à l'aide de critère basés sur la pertinence, la faisabilité et les avantages qu'ils y voyaient. Cela les a amenés à distinguer l'interdépendance des différents types de savoirs convoqués dans les planifications d'activités selon la nouvelle littératie. engager dans des activités de planification pédagogique où ils intégreront les diverses 
facettes de la compétence en littératie multimodale. Ainsi, en créant des vidéos, en intégrant des photos à un texte de fiction ou en se livrant à toute autre activité multimodale, ils se poseront d'utiles questions sur le nécessaire recours aux divers modes sémiotiques pour faire croître chez leurs élèves la compréhension et la production de multitextes. Ils pratiqueront aussi la transmédialisation (le passage d'un mode de représentation sémiotique à un autre, par exemple, la transposition d'un roman en film).

Il est important, une fois la formation enseignante assurée, que le chercheur aille vérifier dans les classes si le développement professionnel se poursuit par la mise en place de réelles activités de littératie médiatique multimodale, qui supposent, par essence, la réciprocité, l'évolution des relations et une réflexion épistémologique sur la diversité des modes sémiotiques, de leur appréhension et de leur utilisation. Il s'agit, en somme, d'une mutation culturelle encore plus que d'une mutation professionnelle.

\section{Conclusion : miser sur une formation de pointe en littératie médiatique multimodale}

28 Même si les chercheurs ont prouvé la rentabilité des pratiques de littératie médiatique multimodale que nous venons d'exposer, il n'est pas dit que les enseignants seront convaincus de leur bien-fondé, qu'ils les essaieront spontanément (et non plus en contexte expérimental) et qu'ils seront suffisamment motivés pour en faire des pratiques quotidiennes. Il est donc très important que la formation initiale des enseignants insiste sur l'apport de la nouvelle littératie, avant que ne s'ancrent des modalités plus conservatrices de transmission du savoir chez les nouveaux maitres.

Il conviendrait aussi que les futurs enseignants entreprennent une réflexion sur les besoins sociaux de leurs élèves dans une société en constante mutation, car la littératie médiatique multimodale est plus qu'une formule, c'est une position socioculturelle basée sur une épistémologie du savoir qui est différente de celle ayant eu cours jusqu'à l'arrivée de l'ordinateur et des recherches sur Internet.

S'ils ne sont pas convaincus à titre personnel de la nécessité de s'adapter en ce sens, peutêtre que la constatation du grand engouement de leurs élèves pour de telles pratiques et les résultats qu'ils obtiennent lorsqu'on les guide dans leurs travaux dans ce domaine les convaincront. Il faut toutefois les accompagner professionnellement, comme les chercheurs du modèle TPACK l'ont fait.

31 Les futurs enseignants doivent, dès leur entrée dans la profession, concevoir qu'ils devront toute leur vie professionnelle s'adapter à des changements sociétaux et culturels. Pour des formateurs de maîtres, engager les futurs enseignants dans pareille démarche, c'est leur permettre de devenir des agents de changement, non seulement dans leur école, mais également dans la communauté éducative en général. Ils surmonteront aussi la rigidité des curricula et feront preuve d'ingéniosité face à un éventuel manque d'équipement. Leur attention aux aspects sociaux et culturels des pratiques de littératie médiatique multimodale, leur désir de "partager le pouvoir " avec leurs élèves seront autant de manifestations du fait que la nouvelle littératie sera devenue pour eux plus qu'un instrument, mais bien l'essence même de l'apprentissage. 


\section{BIBLIOGRAPHIE}

Bailey, N., (2006). Designing social futures: Adolescent literacy in and for new Times. Unpublished doctoral dissertation, University at Buffalo, State University of New York (disponible sur ProQuest).

Boissinot, A. (2010). « La formation des maîtres : débats et perspectives », Revue internationale d'éducation de Sèvres, 55 | décembre. En ligne : http://ries.revues.org/920

Buckingham, D., (2003). «Media Education and the End of the Critical Consumer», Harvard Educational Review, (73) 3, 309-327.

Cope, B. et Kalantzis, M., (2000). «Multiliteracies: The beginning of an idea». in B. Cope, et M. Kalantzis (Eds.), Multiliteracies: Literacy learning and the design of social futures. pp. 3-8. Londres: Routledge.

Douehi, M., (2011). Pour un humanisme numérique. Paris : Seuil.

Durkheim, É., (1912). Les formes élémentaires de la vie religieuse. Le système totémique en Australie. Paris : Presses universitaires de France, 1968, cinquième édition.

Enochsson, A., C. Rizza, OCDE, ICT (2009). in Initial Teacher Training: Research Review, OECD

Education Working Papers, No. 38, OECD Publishing. En ligne : http://

dx.doi.org/10.1787/220502872611

Jewitt, C., (2009). «An Introduction to Multimodality». In C. Jewitt (Ed.) The Routledge Handbook of Multimodal Analysis, Londres, Routledge, 14-27.

Karsenti, T., Peraya, D. et Viens, J., (2002). «Conclusion : bilan et prospectives de la recherche sur la formation des maîtres à l'intégration pédagogique des TIC ». Revue des sciences de l'éducation.

(28) 2, 459- 470 .

Koehler, M. J. et Mishra P., (2005). «What happens when teachers design educational technology? The development of technological pedagogical content knowledge». Journal of educational computing research, (32) 2, 131-152, Baywood.

Kress, G., (2010). Multimodality: a Social Semiotic Approach to Contemporary Communication, New-York: Routledge.

Kress, G., (2009). «What Is Mode? ». In C. Jewitt (Ed.), The Routledge Handbook of Multimodal Analysis, pp54-67, Londres: Routledge

Kress, G., (2003). Literacy in the new media age. New-York: Routledge.

Lankshear, C., et Knobel, M. (2003). New Literacies: Changing knowledge and classroom learning. Philadelphia: Open University Press.

Lebrun, M. Lacelle, N. et Boutin, J-F. (dir.) (2012). La littératie médiatique multimodale. De nouvelles approches en lecture-écriture à l'école et hors de l'école. Sainte-Foy: Presses de l'Université du Québec. $270 \mathrm{p}$.

Le Deuff, O. et Berra , A. (2012). «Quelles compétences et littératies pour les humanités numériques? » THATCamp Paris 2012. Non-Actes de la non-conférence des humanités numériques. Paris: 
Éditions de la Maison des Sciences de l'Homm, En ligne: http://books.openedition.org/ editionsmsh/334

Liu, A. (2012). «Where is cultural criticism in the digital humanities». Debates in the Digital Humanities. En ligne $:$ http://dhdebates.gc.cuny.edu/debates/text/20

Miller, S. (2007) «English Teacher Learning for New Times: Digital Video Composing as Multimodal Literacy Practice», English Education, (40), 61-83. En ligne: http://www.jstor.org/ stable/40173268

Mounier, P. (2011). «Qu'apportent les digital humanities? Quelques exemples ». Homo Numericus, En ligne : http://homo-numericus.net/spip.php?breve1011

New London Group (2000) «A Pedagogy of Multiliteracies: Designing Social Futures». In Cope, B. et Kalantzis, M. (Eds), Multiliteracies: Literacy learning and the design of social futures (pp 9-37) Londres: Routledge.

Rey, V. et Beaudet, C. (2011). « Rapports aux textes virtuels en formation de lettres : vers une nouvelle écriture professionnelle», Lidil, 43, 103-116.

Tabary-Bolka, L. (2009). « Culture adolescente vs culture informationnelle. L'adolescent acteur de la circulation de l'information sur internet », Les Cahiers du numérique, (3) 5, 85-97. En ligne :

http://semiopat.free.fr/artecom/communication/downloads-2/files/culture\%

20informationnelle.pdf

Walsh, M. (2008). «Worlds have collided and modes have merged: Classroom evidence of changed literacy practices». Literacy, (42) 2, 101-108.

\section{RÉSUMÉS}

Les humanités numériques issues de l'essor des nouvelles technologies, entre autres de l'apparition de l'Internet, ont stimulé la croissance de la littératie médiatique multimodale, qui se décline en pratiques extra-scolaires et scolaires. L'article décrit les fondements épistémologiques et empiriques de cette nouvelle littératie et donne quelques exemples de son application à la formation des futurs enseignants de français, plus particulièrement en suivant le modèle TPACK de Koehler et Mishra.

The development of new technologies and of the web has created the digital humanities and has stimulated the growth of multimodal media literacy, which is found in extra-curricular and school practices. The paper describes the epistemological and empirical foundations of this new literacy and gives some examples of its application to the training of future teachers of French, mainly in following the TPACK model of Koehler and Mishra.

\section{INDEX}

Mots-clés : développement professionnel, humanités numériques, littératie médiatique multimodale

Keywords : digital humanities, multimodal media literacy, professional development 


\section{AUTEUR}

\section{MONIQUE LEBRUN}

Université du Québec, Montréal, Équipe de recherche interuniversitaire LITMEDMOD, (UQAMUQTR-UQAR) 